\title{
O PRINCÍPIO DAS GAVETAS DE DIRICHLET
}

\section{THE PRINCIPLE OF DIRICHLET DRAWERS}

Juliano Ferreira de Lima'; Marco Antonio Travassos'; Vanessa de Freitas Travello'; Antonio Carlos Tamarozzi².

${ }^{1}$ Aluno do curso de Lic. em Matemática da UFMS e bolsista do Programa de Educação Tutorial/SESU - Matemática - UFMS - Campus de Três Lagoas;

${ }^{2}$ Professor Associado do curso de Lic. em Matemática da UFMS e tutor do Programa de Educação Tutorial/SESU - Matemática - UFMS - Campus de Três Lagoas;

1juliano.ferreira.info2@gmail.com; 2act.ufms@gmail.com;

RESUMO - O Princípio das gavetas de Dirichlet também conhecido como O Princípio da Casa dos Pombos, pode ser apresentado tanto como um resultado matemático, quanto como um método de prova. Como um resultado matemático, o Princípio das gavetas de Dirichlet é bastante simples e intuitivo e parece, à primeira vista, ser de pouca aplicabilidade. Mas, quando usado como um método de prova, ele se torna uma ferramenta extremamente poderosa na resolução de problemas, como veremos no decorrer desse trabalho. O Princípio das Gavetas de Dirichlet recebeu este nome depois que o matemático alemão Dirichlet usou frequentemente este princípio em seu trabalho, no século XIX.

Palavras-chave: Casa dos pombos; Função; Análise de dados; Contagem e Conjuntos.

ABSTRACT - The Principle of the drawers of Dirichlet's Principle also known as the House of Pigeons, can be presented both as a mathematical result, and as a method of proof. As a mathematical result, the Dirichlet drawer principle is quite simple and intuitive and seems at first sight to be of little applicability. But when used as a method of proof, it becomes an extremely powerful tool in solving problems, as we shall see in the course of this work. The Principle of Drawers Dirichlet is named after the German mathematician Dirichlet often used this principle in his work, in the nineteenth century.

Keywords: House of pigeons; Function; Data Analysis; Sets and Counting. 


\section{INTRODUÇÃO}

No presente trabalho apresentamos o princípio da casa dos pombos, também conhecido como princípio das gavetas ou princípio de Dirichlet. Este princípio matemático baseia-se na afirmação simples de que, se temos que distribuir $\mathrm{n}$ pombos em $n-1$ gaiolas, então ao menos uma das gaiolas conterá, no mínimo, dois pombos. Matematicamente falando, isto é equivalente a dizer que se o número de elementos de um conjunto finito A for maior do que o número de elementos de um outro conjunto $B$, então uma função de $A$ em $B$ não pode ser injetiva. O princípio de Dirichlet pode ser aplicado em muitos problemas formais, incluindo aqueles que envolvem um conjunto infinito. Ele é muito útil para resolver problemas que, pelo menos à primeira vista, não são imediatos e admite consequências surpreendentes, conforme mostram os exemplos que apresentamos neste trabalho. A sua aplicação exige identificar, na situação dada, quem faz o papel dos pombos e quem faz 0 papel das gaiolas.

O princípio de Dirichlet admite generalizações que também possui grande espectro aplicativo. As demonstrações e aplicações podem ser apresentadas de maneira simples, acessível e cativante aos estudantes e constituem o objetivo deste trabalho.

\section{METODOLOGIA}

O trabalho é resultado de uma pesquisa teórica, desenvolvida através de discussões do tema com o orientador $\mathrm{e}$ apresentações de seminários. Teve início como parte das atividades do programa de educação tutorial (PET) - Matemática, sobre estudos de métodos de contagem, dentro da área de Introdução à Combinatória e Aritmética. O trabalho incluiu as etapas de leitura e resoluções de exercícios e a seleção de atividades propostas com aplicações interessantes para propor a estudantes do ensino médio. Como medida para o despertar do interesse no tema, priorizamos problemas com aplicações em situações geométricas.

\section{RESULTADOS}

Suponha que um grupo de 10 pombos voe para dentro de 9 casas para empoleirarem-se. Visto que há 10 pombos e 9 casas, pelo menos uma dessas 9 casas deverão ter no mínimo dois pombos. 0 funcionamento deste processo pode ser atestado com o seguinte argumento. Se cada casa tiver no máximo um pombo, então no máximo 9 pombos estarão acomodados sobrando um pombo. Por esta razão este principio de contagem matemática, recebeu o nome de "Princípio da casa dos pombos".

Vamos visualizar aplicações que evidenciam esta abordagem como um 
método importante de contagem matemática, que ultrapassa este senso comum de pombos e casas.

O Princípio da Casa dos Pombos foi utilizado publicamente, pela primeira vez, pelo matemático alemão G. Lejeune Dirichlet em 1834 com o nome de Schubfachprinzip ("princípio das gavetas"). Em sua homenagem, portanto, ficou conhecido como Princípio das Gavetas de Dirichlet.

Teorema 1. (O Princípio de Dirichlet) Seja $\mathrm{k}$ um número inteiro positivo. Se $\mathrm{k}+$ 1 ou mais objetos são colocados dentro de $\mathrm{k}$ caixas, então há uma caixa que terá dois ou mais objetos.

Demonstração. Vamos demonstrar o princípio da casa dos pombos usando uma demonstração por absurdo. Suponha que nenhuma das $\mathrm{k}$ caixas contenha mais de um objeto. Então, a contagem de no máximo um objeto em cada uma das k caixas, fornece um total de no máximo k objetos, o que é uma contradição, porque há pelo menos $\mathrm{k}+1$ objetos.

O princípio da casa dos pombos pode ser utilizado para demonstrar resultados puramente matemáticos, como o seguinte. Se o número de elementos de um conjunto finito A é maior que o número de elementos de outro conjunto $B$, então uma função de $A$ em B não pode ser injetiva, como observamos no corolário a seguir.
Corolário. Uma função $\mathrm{f}$ de um conjunto $\mathrm{k}+1$ ou mais elementos para um conjunto com k elementos não é injetiva.

Demonstração. Suponhamos que para cada elemento $\mathrm{y}$, no contradomínio de $\mathrm{f}$ temos uma caixa que contém todos os elementos $\mathrm{x}$ do domínio de $\mathrm{f}$, tal que $\mathrm{f}(\mathrm{x})=\mathrm{y}$. Como o domínio contém $\mathrm{k}+1$ ou mais elementos e o contradomínio contém apenas k elementos, o princípio da casa dos pombos nos diz que uma das caixas contém dois ou mais elementos $\mathrm{x}$ do domínio. Isso significa que $f$ não pode ser uma função injetora.

Exemplo 1. Quantos alunos deve haver em uma sala para podermos afirmar que pelo menos dois estudantes tenham a mesma nota em uma determinada prova, se a nota é graduada em um número inteiro de 0 a 10 ?

Solução: De 0 a 10 existem 11 números possíveis. O princípio de Dirichlet mostra que entre 12 estudantes há pelo menos dois com a mesma nota.

O princípio da casa dos pombos afirma que deverá haver pelo menos dois objetos na mesma caixa quando existirem mais objetos que caixas. Desta forma, o princípio de Dirichlet, pode ser generalizado da forma seguinte, onde, o símbolo $\lceil\mathrm{m}\rceil$ representa o maior inteiro menor ou igual a m. 
Teorema 2. (A Generalização do Princípio de Dirichlet). Se $\mathrm{n}$ objetos são colocados em k caixas, então há pelo menos uma caixa com $[\mathrm{n} / \mathrm{k}\rceil$ objetos.

Demonstração. Faremos uma demonstração por absurdo.

Suponha que nenhuma das caixas tenha mais que $[\mathrm{n} / \mathrm{k}\rceil-1$ objetos. Então, o número total de objetos é no máximo

$$
\mathrm{k}\left(\left\lceil\frac{\mathrm{n}}{\mathrm{k}}\right\rceil-1\right)<\mathrm{k}\left(\left(\frac{\mathrm{n}}{\mathrm{k}}+1\right)-1\right)=\mathrm{n}
$$

em que a inequação $[\mathrm{n} / \mathrm{k}\rceil<(\mathrm{n} / \mathrm{k})+1$ foi usada. Isto é um absurdo, pois há um total de $\mathrm{n}$ objetos. Portanto, existe ao menos uma caixa com $[\mathrm{n} / \mathrm{k}]$ objetos.

Existem inúmeros problemas que avaliam o número mínimo de objetos, de tal forma que pelo menos $r$ desses objetos deverão estar uma distribuídos em $\mathrm{k}$ caixas quando esses objetos forem distribuídos entre as caixas. Assim, percebemos trivialmente que se tivermos $\mathrm{n}$ objetos, a generalização do princípio da casa dos pombos afirma que existem pelo menos $r$ objetos em uma das caixas, desde que $\lceil\mathrm{n} / \mathrm{k}\rceil \geq \mathrm{r}$.

Apresentamos a seguir, uma sequencia de exemplos que utiliza o Principio de Dirichlet nas duas formas, clássica e extendida.

Exemplo 2. Entre 500 pessoas pelo menos quantas nasceram no mesmo mês?
Solução: Utilizando o teorema de Dirichlet vemos que: $\lceil 500 / 12\rceil=42$, assim podemos afirmar que pelo menos 42 pessoas nasceram no mesmo mês.

Exemplo 3. Em uma gaveta há 6 pares de meias brancas e 6 pares de meias pretas. Quantas meias deverão ser retiradas ao acaso para termos certeza de obter um par de meias da mesma cor?

Solução: Pensando nas meias como pombos e nas duas cores como as duas casas, vemos que com 3 meias haverá duas meias com a mesma cor.

Exemplo 4. João convidou 49 amigos para sua festa de aniversário. Podemos afirmar que em sua festa existiam pelo menos:

a) 5 pessoas que fazem aniversario no mesmo mês ?

b) 8 pessoas que nasceram no mesmo ano?

c) 6 pessoas que nasceram no mesmo dia da semana?

d) 2 pessoas que nasceram no mês de janeiro?

\section{Solução:}

a) Verdadeira.

$\mathrm{O}$ ano tem 12 meses e podemos considerar cada mês como uma casa. Assim, $n=12$. Pelo princípio da casa dos pombos, vemos:

$$
\left[\frac{49}{12}\right]+1=5
$$

b) Falsa. 
O numero 49 não é suficientemente grande para podermos assegurar a afirmação, diante do número de anos que os convidados podem ter nascido.

c) Verdadeira.

A semana tem 7 dias (domingo, segunda, terça, ..., sábado), assim, a afirmação é verdadeira pois

$$
\left[\frac{49}{7}\right]=7
$$

d) Falsa.

Pelo item (a) podemos garantir que pelo menos um mês em que pelo menos 5 pessoas nasceram, mas o princípio da casa dos pombos, não assegura qual é o mês.

Exemplo 5. Qual o menor número de alunos necessário em uma sala de aula para podermos afirmar que pelo menos quatro receberão a mesma nota, se são possíveis cinco notas: $\mathrm{n}_{1}, \mathrm{n}_{2}, \mathrm{n}_{3}, \mathrm{n}_{4}, \mathrm{n}_{5}$.

Solução: $\mathrm{O}$ número mínimo $\mathrm{n}$ de estudantes necessários para garantir que pelo menos quatro alunos tirem a mesma nota é o menor número inteiro $\mathrm{n}$ tal que $\lceil\mathrm{n} / 3\rceil=4$. Através da generalização do princípio da casa dos pombos vemos que o menor número inteiro é $\mathrm{N}=10$. Notemos que se tivermos apenas 9 estudantes, é possível que haja três que receberão cada nota, e não quatro. Assim, 10 é o número procurado.

Exemplo 6. Escolhem-se 5 pontos ao acaso sobre a superfície de um quadrado de lado 2. Mostremos que pelo menos um dos segmentos que eles determinam tem comprimento menor ou igual a $\sqrt[2]{2}$.

Solução: Neste caso, está claro que os objetos são os 5 pontos.

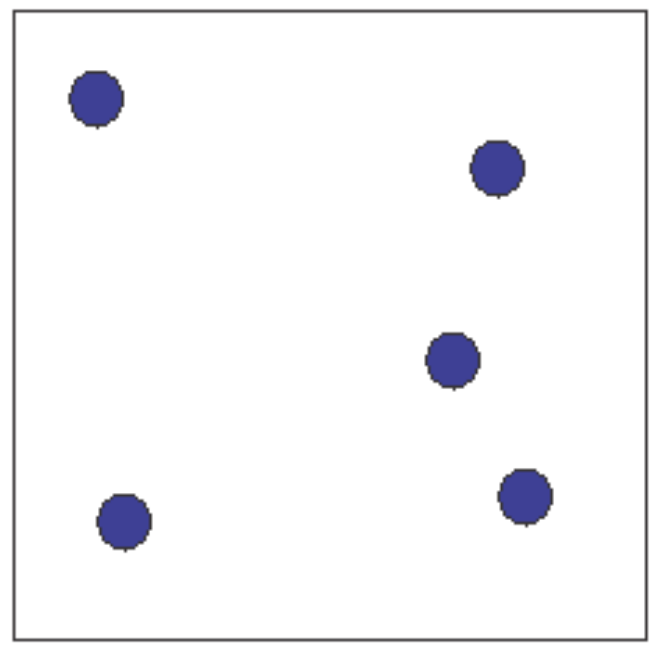

Figura 1. Ilustração do enunciado.

O ponto chave da resolução está na identificação das gavetas. Devemos subdividir o quadrado dado em 4 partes de modo tal que a distância entre dois pontos situados em uma destas partes nunca seja maior que $\sqrt[2]{2}$. (Figura2).

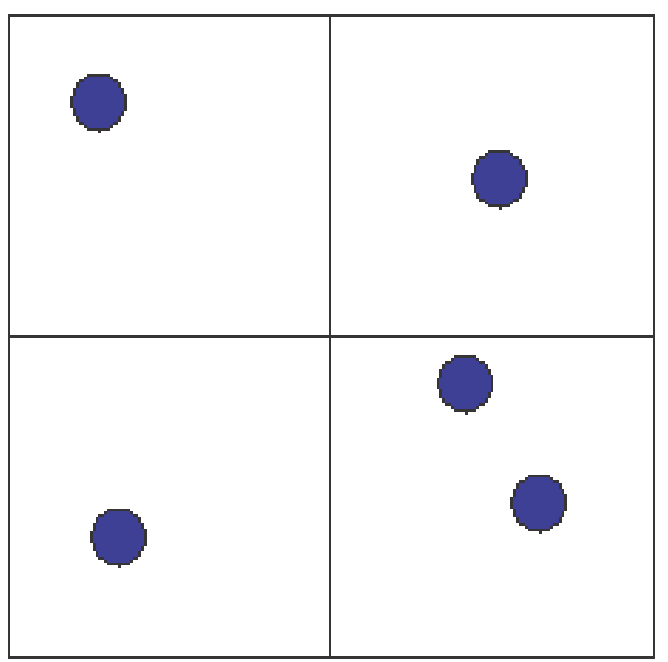

Figura 2. Resolvendo o enunciado. 
Basta dividi-lo nos quatro quadrados determinados pelas retas que unem os pontos médios dos lados opostos.

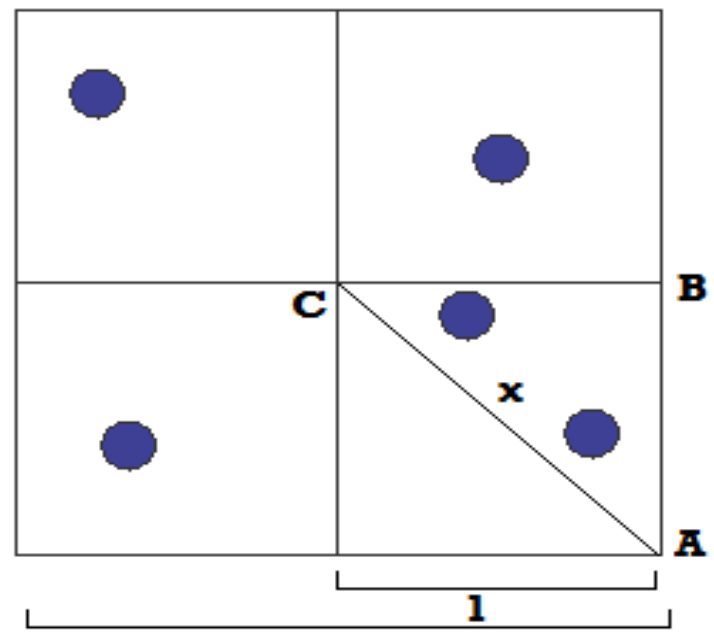

2

Figura 3. Explorando o enunciado.

Utilizando o Teorema de Pitágoras no Triângulo $A B C$, vemos que $x=\sqrt[2]{2}$, logo, os dois pontos que estão dentro da mesma gaveta não podem ter comprimento maior que $\sqrt[2]{2}$

Assim, cada uma destas quatro gavetas, a distância máxima entre dois pontos é igual à sua diagonal, que mede 2 . Portanto, dados 5 pontos, pelo menos 2 estarão em uma mesma "gaveta" e, assim, determinam um segmento de comprimento menor ou igual $a \sqrt[2]{2}$.

Exemplo 7. Todos os pontos de um plano são pintados de azul ou vermelho. Provemos que é possível encontrar dois pontos da mesma cor que distam exatamente $10 \mathrm{~cm}$.
Solução: Por definição, num plano existem infinitos pontos que estão contidos nele e estes, supostamente podem ser divididos em azul e vermelho.

Imaginemos neste plano um triângulo equilátero de lado igual a $10 \mathrm{~cm}$, conforme a figura seguinte

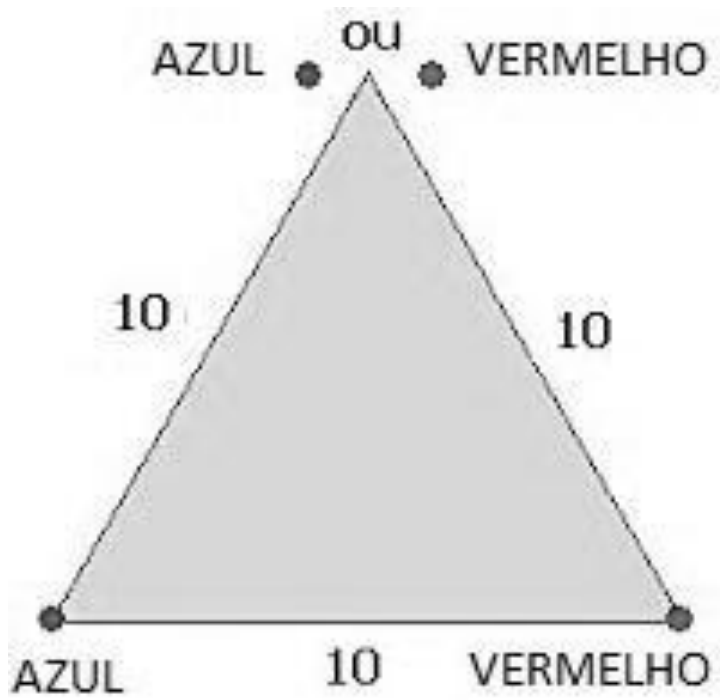

Figura 4. Triângulo equilátero.

Como são duas cores (casas) e três pontos (pombos). Pelo princípio da casa dos pombos teremos dois pontos da mesma cor.

Exemplo 8. Consideremos um quadrado com os lados medindo 3u.c. $\mathrm{Na}$ superfície quadrangular por ele determinada, marquemos aleatoriamente 10 pontos. Mostre que pelo menos um dos segmentos determinados por esses pontos tem comprimento menor que ou igual a $\sqrt{2}$ u.c. (u.c. significa unidade de comprimento).

Solução: Consideremos um quadrado de lado igual a 3.u.c, em seguida, vamos 
dividi-lo em 9 quadrados menores lado igual à 1.u.c.

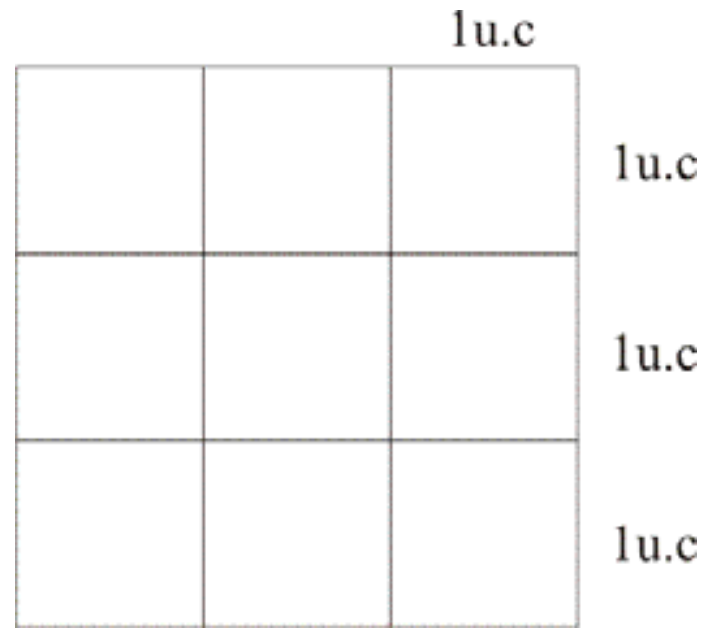

Figura 5. Quadrado de lado 3u.c.

Ao considerarmos os quadradinhos como casas e os pontos como pombos, a quantidade de casas será 9 que é menor que a quantidade de pombos 10 , assim, pelo Princípio da Casas dos Pombos, pelo menos, um quadrado possuirá 2 pontos.

Consideremos o triângulo retângulo $A B C$.

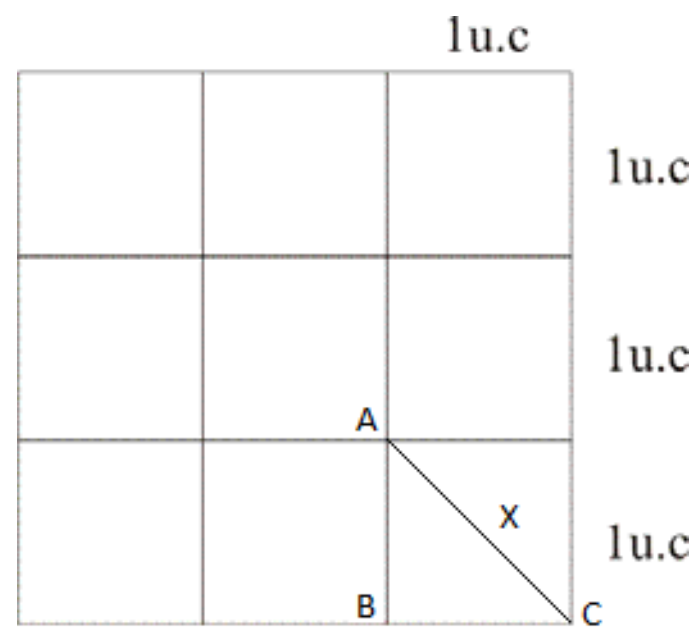

Figura 6. Quadrado de lado 1u.c.

Utilizando o Teorema de Pitágoras no Triângulo $A B C$, vemos que $X=\sqrt{2} u . c$, logo, existirão, pelo menos, dois pontos cujo segmento por eles determinado mede no máximo $\sqrt{2}$ u.c.

Exemplo 9. Escolhem-se nove pontos no interior de um quadrado de lado 1 u.c. Mostrar que é possível escolher três pontos de tal forma que a área do triângulo que formam é menor ou igual a $\frac{1}{8}$.

Solução: Sabemos que os nove pontos estão contidos no quadrado de lado 1 . Consideremos um quadrado $A B C D$ e $M, N, O$ e $P$ pontos médios do quadrado como mostra a figura a seguir.

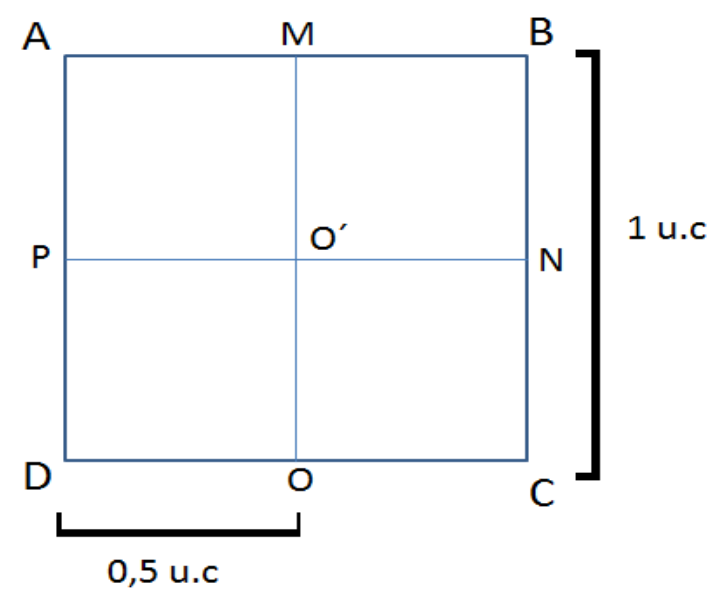

Figura 7. Quadrado de lado 1u.c.

Notemos que temos 9 pontos (nove pombos) e 4 quadrados menores (quadro casas), assim, pelo princípio da casa dos pombos, em um quadrado terá pelo menos 3 pontos.

Suponhamos que os três pontos estejam no quadrado $A M P O^{\prime}$, logo, a maior distância entre eles pode ser apenas $\frac{1}{2}$ u.c, ou seja, apenas no caso dos pontos serem exatamente os vértices do quadrado. 
Consideremos o triângulo formado por $A P M$.

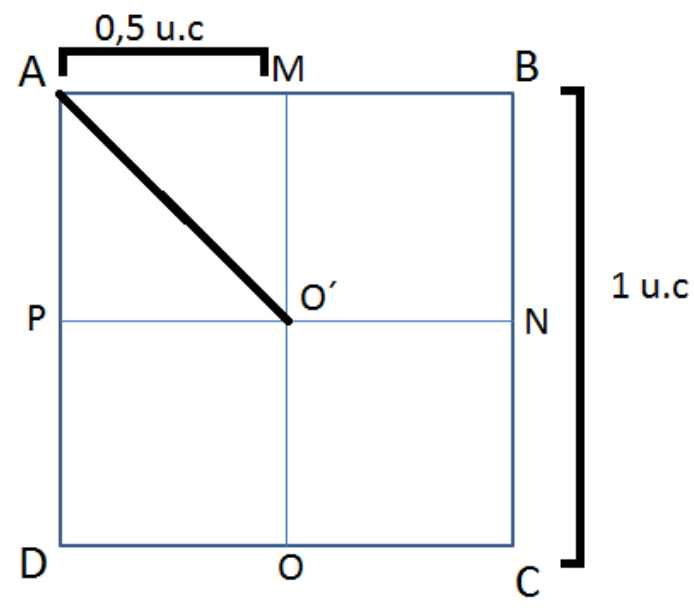

Figura 8. Triângulo isósceles de lado

1u.c.

No triângulo $A P O^{\prime}$ temos:

$$
\begin{gathered}
\operatorname{Area}_{A P O^{\prime}}=\frac{\overline{A P} \cdot \overline{P O^{\prime}}}{2} \Rightarrow \operatorname{Area}_{A P O^{\prime}}=\frac{\frac{1}{2} \cdot \frac{1}{2}}{2} \\
\operatorname{Area}_{A P O^{\prime}}=\frac{1}{8} .
\end{gathered}
$$

De onde segue a afirmação.

Exemplo 10. Mostremos que entre quaisquer $\mathrm{n}+1$ números inteiros positivos não excedentes a $2 \mathrm{n}$, deverá haver um número inteiro que divide um dos outros inteiros.

Solução: Podemos escrever cada um dos $\mathrm{n}+1$ números inteiros $\mathrm{a}_{1}, \mathrm{a}_{2}, \ldots, \mathrm{a}_{\mathrm{n}+1}$ como um produto de uma potência de 2 por um inteiro ímpar. Em outras palavras, temos $a_{j}=2^{k_{j}} q_{j}$ para $j=1,2, \ldots, n+1$, em que $k_{j}$ é um número inteiro não negativo (possivelmente nulo) e $\mathrm{q}_{\mathrm{j}}$ é ímpar. Os inteiros $\mathrm{q}_{1}, \mathrm{q}_{2}, \ldots, \mathrm{q}_{\mathrm{n}+1}$ são todos números inteiros positivos ímpares menores que $2 \mathrm{n}$. Visto que há apenas $\mathrm{n}$ inteiros positivos ímpares menores que $2 n$, podemos afirmar, a partir do princípio da casa dos pombos, que dois dos inteiros $\mathrm{q}_{1}, \mathrm{q}_{2}, \ldots, \mathrm{q}_{\mathrm{n}+1}$ deverão ser iguais. Então, há números inteiros i e j, tal que $\mathrm{q}_{\mathrm{i}}=\mathrm{q}_{\mathrm{j}}$. Considere $\mathrm{q}$ como $\mathrm{o}$ valor comum de $\mathrm{q}_{\mathrm{i}}$ e $\mathrm{q}_{\mathrm{j}}$. Assim, $\mathrm{a}_{\mathrm{i}}=2^{\mathrm{k}_{\mathrm{i}}} \mathrm{q}$ e $\mathrm{a}_{\mathrm{j}}=2^{\mathrm{k}_{\mathrm{j}}} \mathrm{q}$. Concluímos que se $\mathrm{k}_{\mathrm{i}}<\mathrm{k}_{\mathrm{j}}$, então $\mathrm{a}_{\mathrm{i}}$ divide $\mathrm{a}_{\mathrm{j}}$; enquanto e $\mathrm{k}_{\mathrm{i}}>\mathrm{k}_{\mathrm{j}}$, então $\mathrm{a}_{\mathrm{j}}$ divide $\mathrm{a}_{\mathrm{i}}$.

\section{DISCUSSÃO}

Alguns resultados e afirmações na matemática tornam-se atraentes porque exploram relações entre conjuntos finitos e são expressos em uma linguagem coloquial. Parte desta atratividade vem justamente do fato de que podem ser formulados simplificadamente, e, não obstante, muitas vezes, a partir deles, diversos problemas são resolvidos, sem recorrer a fórmulas ou a técnicas complicadas.

O Principio de Dirichlet apresentado neste trabalho, descreve esta situação. Com uma formulação simplificada e de grande facilidade de assimilação, o referido tem condições de resolver problemas de impacto, dentro e fora da Matemática.

\section{CONSIDERAÇÕES FINAIS}

O trabalho é resultado de uma pesquisa teórica, desenvolvida através de discussões do tema com o orientador e 
apresentações de seminários como parte das atividades do Programa de Educação Tutorial (PET) - Matemática (UFMS/CPTL), no estudo de Introdução à Combinatória e Aritmética. 0 trabalho incluiu uma etapa de leitura e resoluções de exercícios, desenvolvimento das atividades propostas e a investigação dos resultados obtidos. $\mathrm{O}$ estudo e as atividades desenvolvidas foram avaliados através da apresentação de seminários de discussão.

Como resultado deste trabalho apresentamos a proposta de inserir 0 Princípio da Casa dos Pombos no ensino básico, como recurso para despertar o interesse do estudo pela Matemática. Com efeito, trata-se de um assunto acessível, para o qual podem ser elaboradas sequencias de problemas interessantes e relevantes para o cotidiano dos estudantes. De maneira gradual pode atingir um grau sofisticado de generalização do tema, de modo a aperfeiçoar competências de investigação, organização e comunicação da informação.

\section{AGRADECIMENTOS}

Os autores agradecem ao FNDE Fundo Nacional de Desenvolvimento da Educação, pelo suporte financeiro concedido ao PET - Matemática (UFMS/CPTL), no desenvolvimento das atividades de ensino, pesquisa e extensão, que possibilitaram este trabalho.

\section{REFERÊNCIAS}

LIMA, Elon Lages. Número e Funções Reais / Elon Lages Lima. Rio de Janeiro: SBM, 2013. 297p. (Coleção PROFMAT, 07).

LIMA, Elon Lages. A Matemática do Ensino Médio / Elon Lages Lima. Rio de Janeiro: SBM, 1999. (Coleção do Professor de Matemática).

MARTINEZ, Fabio Brochero; et al. Teoria dos números: um passeio com primos e outros números familiares pelo mundo inteiro / Fabio Brochero Martinez; et al. 2 ed. Rio de Janeiro: IMPA, 2013, 481 P. (Projeto Euclides).

MORGADO, Augusto César de Oliveira; CARVALHO, João Bosco Pitombeira; CARVALHO, Paulo Cezar Pinto; FERNANDEZ, Pedro. Análise Combinatória e Probabilidade com as soluções dos exercícios. Coleção do Professor de Matemática. Nona edição. Rio de Janeiro: Sociedade Brasileira de Matemática, 2006.

MUNIZ NETO,Antonio Caminha. Tópicos de Matemática Elementar: combinatória / Caminha Muniz Neto. -1. ed. Rio de janeiro: SBM, 2012.

ROSEN, Kenneth H. Matemática discreta e suas aplicações / Kenneth H. Rosen; [tradução João Giudice]. São Paulo: McGrawHill, 2009.

SCHEINERMAN, Eduard R. Matemática Discreta: uma introdução/ Thomson Learning, 2006. 